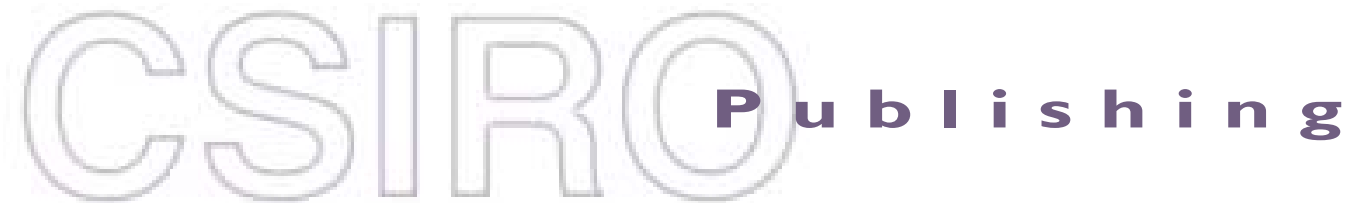

\section{Publications of the Astronomical Society of Australia}

Volume 19, 2002

(C) Astronomical Society of Australia 2002

An international journal of astronomy and astrophysics

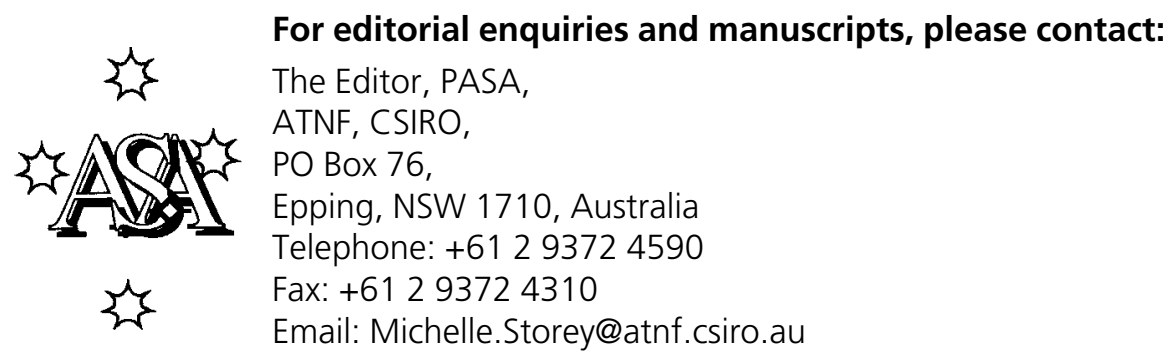

For general enquiries and subscriptions, please contact: CSIRO Publishing PO Box 1139 (150 Oxford St)

Collingwood, Vic. 3066, Australia

Telephone: +6139662 7666

Fax: +61 396627555

Email: publishing.pasa@csiro.au

C S I RO

PUBLISHING Published by CSIRO Publishing

for the Astronomical Society of Australia

www.publish.csiro.au/journals/pasa 


\title{
Image Processing for the Sydney University Molonglo Sky Survey (SUMSS)
}

\author{
B. H. P. Chan and L. E. Cram \\ School of Physics, University of Sydney, NSW 2006, Australia \\ bchan, lc@physics.usyd.edu.au \\ Received 2001 March 2, accepted 2001 October 9
}

\begin{abstract}
The Sydney University Molonglo Sky Survey (SUMSS) is a deep radio survey at $843 \mathrm{MHz}$, covering the region south of $-30^{\circ}$ declination. Designed to be a southern counterpart of the northern NRAO VLA Sky Survey (NVSS), SUMSS is over 40\% complete, and it is now time to devise ways to generate the source catalogue for the survey. We describe here new methods to deal with image artifacts to minimise spurious fits by automatic source fitting algorithms. With the new techniques, an automatically generated, objective catalogue can be made to a $10 \mathrm{mJy}$ cutoff. Catalogues can be made to a $5 \mathrm{mJy}$ cutoff provided that special care is taken and certain artifacts are avoided.
\end{abstract}

Keyword: techniques: image processing

\section{Introduction}

The Sydney University Molonglo Sky Survey (SUMSS) is a deep radio imaging survey covering $8,000 \mathrm{deg}^{2}$ at $843 \mathrm{MHz}$ (Bock, Large, \& Sadler 1999). SUMSS will be the only radio survey with arcsecond positional accuracy and milli-Jansky sensitivity to cover completely the region south of $-40^{\circ}$ declination. The survey began in June 1997, using the Molonglo Observatory Synthesis Telescope (MOST), operating in the wide-field mode (for a technical description of the MOST see Mills, Little, \& Joss 1976; Robertson 1991; Large et al. 1994). Table 1 lists some specifications of MOST in the wide-field mode. The beam size and sensitivity are similar to those of the northern NRAO VLA Sky Survey (NVSS). When SUMSS is complete, the two surveys together will cover the entire radio sky with quite uniform properties.

SUMSS was $44 \%$ complete in November 2000, and at an imaging rate of $>1000 \mathrm{deg}^{2}$ per year, it is expected to finish late in 2004. The main published image database for SUMSS will comprise $4^{\circ} \times 4^{\circ}$ mosaics, on a grid corresponding to that employed by the NVSS. It is planned to publish in addition a list of radio source parameters such as central position, integrated flux density, and angular size. These source parameters will be obtained by fitting

Table 1. Specifications of the wide-field mode of MOST

\begin{tabular}{ll}
\hline Centre frequency & $843 \mathrm{MHz}$ \\
Bandwidth & $3 \mathrm{MHz}$ \\
Polarisation & Right Hand Circular (IEEE) \\
Declination range* & $-90^{\circ}$ to $-30^{\circ}$ \\
Synthesised beam $(\alpha \times \delta)$ & $43^{\prime \prime} \times 43^{\prime \prime} \operatorname{cosec}|\delta|$ \\
Field size & $164^{\prime} \times 164^{\prime} \operatorname{cosec}|\delta|$ \\
Effective noise after $12 \mathrm{~h}$ & $0.5-1 \mathrm{mJy} / \mathrm{beam}(1 \sigma)$ \\
Surface brightness sensitivity & $0.4-0.9 \mathrm{~K}(1 \sigma)$ \\
Dynamic range (typical) & $100: 1$ \\
\hline
\end{tabular}

*For full hour angle coverage. elliptical Gaussians to sources in the mosaics. There are many different source fitting software packages available; VSAD (a task in AIPS) has several advantages and is at the moment our preferred option for characterising sources.

The MOST produces very high quality synthesised images due to the continuous $\mathrm{u}-\mathrm{v}$ coverage from $40 \lambda$ (set by the $15 \mathrm{~m}$ interarm spacing) to $4.4 \mathrm{k} \lambda$, see Figure 5 in Bock et al. (1999).

Unfortunately but inevitably, artifacts in the images may create spurious sources when fitted automatically, requiring time consuming inspections to remove them. Since the SUMSS source list will include about 300,000 objects at a $5 \mathrm{mJy}$ cutoff, it is important to automate the procedures for ensuring a reliable source catalogue. There are two ways to deal with spurious source fits: (1) remove the artifacts from images before source fitting (described in this paper); (2) develop a computer based artificial intelligence algorithm to flag the spurious sources based on the fitted properties (T. Murphy et al., in preparation). Both methods may be required to optimise the generation of a reliable source list.

This paper discusses two separate aspects of preparing a source list for SUMSS. First we comment on source fitting programs that have been tested for generating the SUMSS source list (Section 2) and explain why we prefer the AIPS task VSAD. Then we describe the common artifacts found in SUMSS images, and explain the methods used to remove them. Finally, we demonstrate the effectiveness of the algorithm with an example.

\section{Source Fitting}

Source fitting procedures are generally divided into two steps. (1) Locating candidate sources: The program searches through the pixel field, seeking groups of pixels above some given cutoff level and locating pixels corresponding to the maxima in the group. The position and peak flux density can then be roughly estimated and an 
estimate of the source size found. (2) Fitting the candidate source: With these initial parameters, the fitting program can perform a least squares fit to minimise the variance between one or more fitted Gaussians and the pixel field, and report refined values and uncertainties for the fitted parameters.

We have tested several source fitting packages (within MIRIAD: IMSAD, QUICKFIT ${ }^{1}$ and SFIND; within AIPS: VSAD ${ }^{2}$ ), to explore differences between fitted parameters when applied to a range of real radio source types. For unresolved and slightly resolved sources, the fitted positional discrepancies between different algorithms are usually under $0.2-0.5$ arcsec, and the fitted flux densities are within $\sim 5 \%$. For extended ( $>3 \times$ beam size) and overlapping sources, the fitted parameters may display large discrepancies between packages, mainly because most fitting packages are incapable of de-blending complex sources (for example by fitting multiple Gaussians). In the light of our studies, we have chosen VSAD since it produces the most consistent performance over the range of tested source types, including multiple Gaussian fits.

\section{Artifacts in SUMSS}

Having selected VSAD as the source fitting program for characterising SUMSS sources, we proceed to describe the problem of image artifacts.

There are three common types of artifacts in MOST observations as shown in Figure 1.

1. Banding: Bright and dark parallel bands sometimes appear across the image, corresponding to structured changes in the base level. They are due to intense radiation from the Sun or ground-based radio transmitters occurring for a short time during the observation.

2. Grating responses: The strongest appear as $4.6^{\circ} \times$ $4.6^{\circ} \operatorname{cosec}|\delta|$ ellipses centred on the source responsible for exciting the response. They are a consequence of the periodic structure of MOST.

3. Radial artifacts: Radial structures are sometimes observed, apparently emerging from strong sources. Their origin is uncertain, but they are most likely caused by small phase and amplitude errors that vary with time, due to the combination of propagation errors in the terrestrial ionosphere and troposphere, and instrumental instabilities.

Another common type of image artifact could be induced after editing to remove bad samples: sidelobes of off-field sources can then extend into the field of view. Figure 2 shows the same SUMSS field as in Figure 1, but with samples affected by interference and the grating response removed. Indicated by boxes are the sidelobes of off-field sources.

\footnotetext{
${ }^{1}$ QUICKFIT was written by Vincent McIntyre. It is a SHELL wrapper to the MIRIAD task IMFIT.

${ }^{2}$ VSAD is a version of SAD modified to generate the preferred NVSS source list.
}

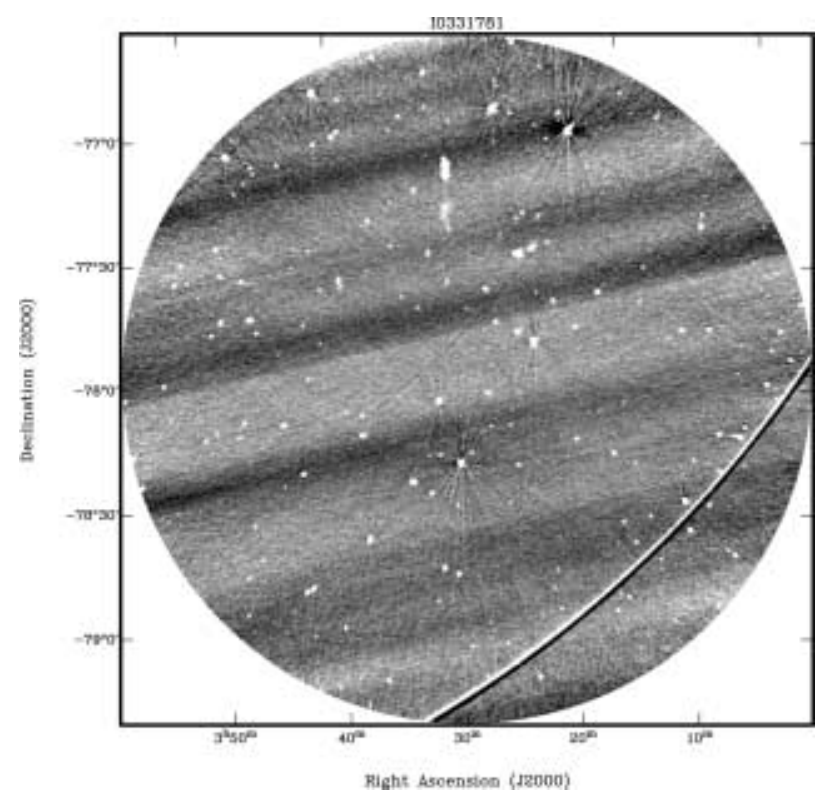

Figure 1 A SUMSS field (I0331781) showing different types of artifacts. This is the CLEANed image produced with all collected samples. There are three types of artifacts in this image: banding (see Section 3.1), grating response (see Section 3.2), and radial artifacts (see Section 3.3). The greyscale is set to -10 to $10 \mathrm{mJy} / \mathrm{beam}$.

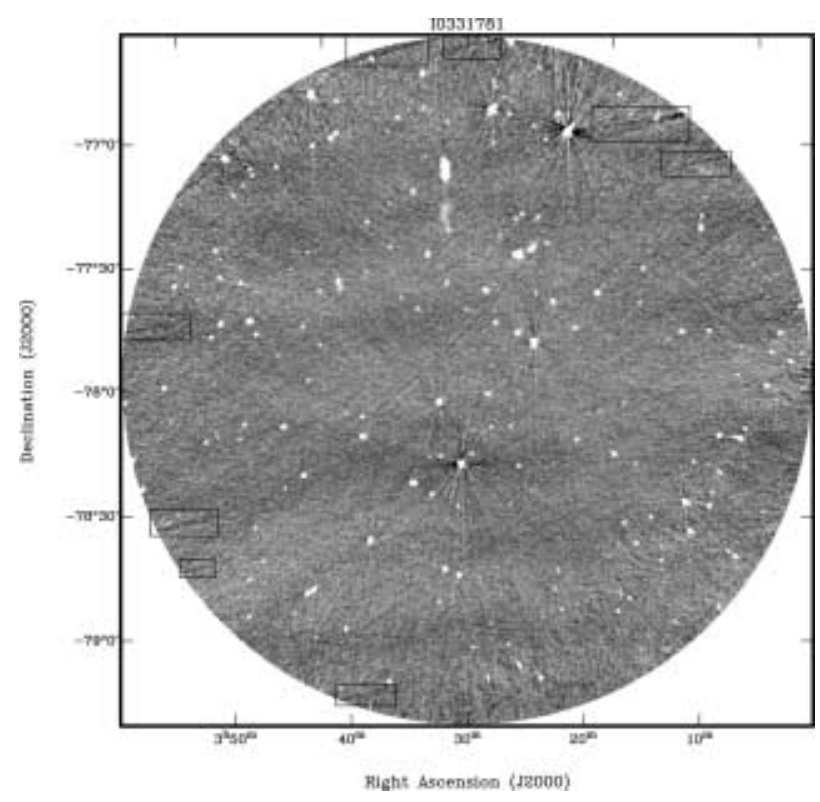

Figure 2 The same SUMSS field (I0331781) as in Figure 1, but with samples affected by interference and the grating response removed (see Section 4). Marked with boxes are areas affected by sidelobes of off-field sources (see Section 3.4). The greyscale is set to -10 to $10 \mathrm{mJy} / \mathrm{beam}$.

\subsection{Solar and Artificial Interference}

The Sun is an extremely strong radio source at $843 \mathrm{MHz}$. Solar radiation can create severe interference during the daytime if the Sun is positioned in a sidelobe of the telescope response to excite the interferometer beams. The requirement for $12 \mathrm{~h}$ to complete a full synthesis means that almost all MOST observations made during the 


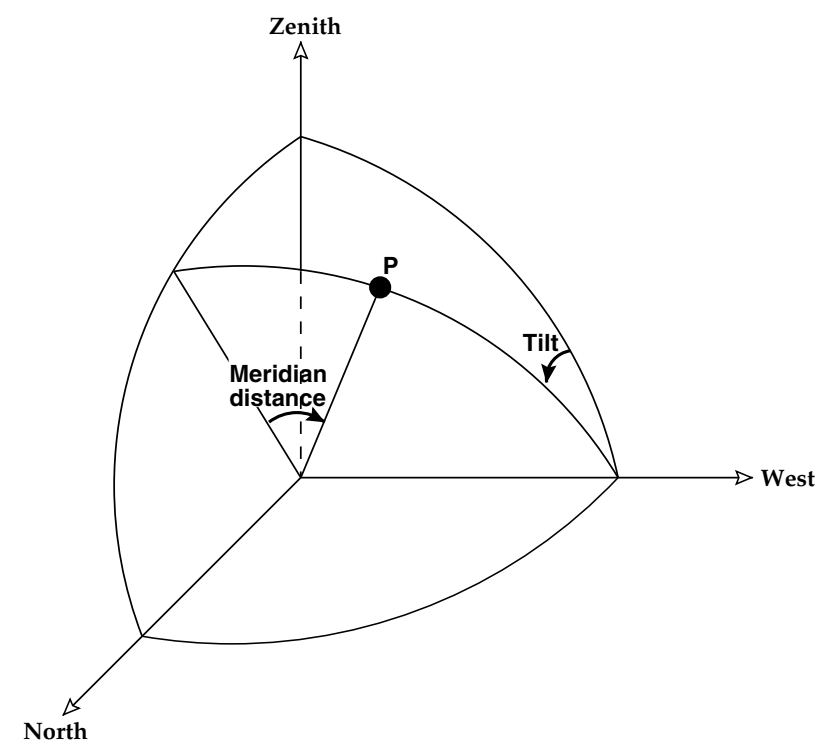

Figure 3 (From Figure 1 of Bock et al. 1999) Coordinate system used with the MOST. The position, $\mathrm{P}$, of a MOST beam is described by a right-handed spherical coordinate system, with the pole pointing west. Tilt is measured toward north from the vertical. Meridian distance $M$ is measured toward west from the meridian plane; it is related to hour angle $H$ and the declination $\delta$, and an approximate relationship is $\sin M=\cos \delta \sin H$.

summer months contain some level of solar interference for a short period.

Owing to geometric delay decorrelation, the MOST beam response away from the pointing centre is sufficiently small that even the Sun does not cause significant interference at most times. However, there are certain positions relative to the pointing centre where sidelobes are higher than average and the Sun can more easily affect the observation. The main types of such sidelobes are (1) symmetry plane, when the tilt (see Figure 3, the same as Figure 1 in Bock et al. 1999) of the Sun equals that of the pointing centre; (2) cone and back beam, when the meridian distances (see Figure 3 ) of the Sun and the pointing centre are the same, or opposite, respectively; and (3) endfire, when the Sun passes close to the east-west axis (meridian distance $= \pm 90^{\circ}$ ). Solar interference is usually confined to a relatively short time interval, and produces parallel bright and dark low spatial frequency banding across the field, as displayed in Figure 1. Because the base level is offset, sources lying in areas affected by banding may not have correct flux densities.

Another source of strong interference to MOST is from ground-based transmitters. Operating at $843 \mathrm{MHz}$, the MOST frequency band is not in an internationally protected radio astronomy band, and it has experienced short bursts of radio interference. In 1996-1997, following joint field trials with the Australian Spectrum Management Agency, local protection of the MOST band was guaranteed until 2006. Artificial interference spanning a relatively short interval can be removed by excluding the corrupted samples, without greatly impairing the quality of CLEANed images, since the dirty beam allows for the deleted samples. If it is particularly important to retain all samples, we have also devised a method for fitting and removing a low order polynomial surface to the base level in an image.

\subsection{Grating Responses}

Strong, regular sidelobes, or grating responses, are produced by an interferometer when there is marked regularity in the distributions of the sampling points. The strongest grating responses (diffraction maxima) occur at angular distances $\theta_{n}$ from a source given by the diffraction equation, $\sin \left(\theta_{n}\right)=n \lambda / D$. Here $\lambda$ is the observing wavelength $(0.356 \mathrm{~m}$ for MOST $)$, and $D$ is the shortest distance between elements. The value of $D$ for MOST in the widefield mode is $4.4 \mathrm{~m}$, resulting in a strong response at an angular offset of $4.6^{\circ}$.

Although grating responses to compact sources have a well defined radius, the amplitude varies with azimuthal position around the source owing to the complex interplay between the distant sidelobes of the synthesised beam and the antenna pattern of the individual $4.4 \mathrm{~m}$ elements. This amplitude variation is difficult to predict, and may attain brightness of $0.5 \%$ of the exciting source in certain parts of the field. Since many SUMSS fields contain sources brighter than $\sim 2 \mathrm{Jy}$, the grating responses are often $\geq 10 \mathrm{mJy}$ and can compromise an automatically generated source list. A strong grating response is present across the bottom right in Figure 1.

Grating responses can be removed by discarding samples taken when the grating response enters the beams. When a grating response is present for a significant portion of an observation (say, $>15 \%$ ) the image quality is reduced dramatically by discarding samples in this way. However, as we explain below, further image processing steps will recover the image quality of areas away from the grating response.

\subsection{Radial Artifacts}

Radial artifacts are due to small, random, time dependent phase variations on a time scale of minutes, arising from instability of the local oscillator and the atmosphere/ ionosphere (Bock et al. 1999). This artifact can be seen in Figures 1 and 2, where symmetric and asymmetric radial structures appear to emerge from strong sources. This type of artifact reduces the dynamic range in the proximity of any strong source. While the strength of these artifacts changes with radial distance from the source, they typically have a strength of $0.5 \%$ close to the central source, so that the image noise approximately doubles around sources of peak flux density $\sim 350 \mathrm{mJy}$. The noise increases over twenty-fold around sources of peak flux density $\sim 5 \mathrm{Jy}$. The radial extent of the spokes is typically $\sim 10^{\prime}$, extending to well over $1^{\circ}$ for strong sources ( $>5 \mathrm{Jy}$ ). At the present stage, radial artifacts may be ameliorated but not totally removed by a self-calibration technique called adaptive deconvolution (Cram \& Ye 1995). 


\subsection{Sidelobes of Off-field Sources}

For fully synthesised MOST images, the point spread function (dirty beam) is compact and azimuthally symmetric. However, if samples are discarded over a range of hour angles (to remove interference or grating responses), the distant sidelobes of the point spread function are enhanced along specific radial directions. These distant sidelobes can be readily removed by CLEANing, provided that the target source lies within the image. However, if the source lies outside the image, the sidelobes may then extend into the field of view and not be CLEANable.

This type of artifact can be removed either by removing all off-field sources before synthesis, or masking the affected area before mosaicing. Both methods have certain technical difficulties.

\section{Removing Artifacts}

MOST raw data acquired in a normal $12 \mathrm{~h}$ observation of a 160 arcmin field comprise 1536 time ordered samples, each representing 7 time multiplexed pointings of $4 \mathrm{sec}$ integration each. A sample contains 896 real-time fan beam responses, separated by 11 arcsec on the sky. The automated image processing pipeline developed for MOST observations is able to identify and eliminate certain classes of defective samples, particularly those affected by strong interference. However, for an optimal image, a certain amount of additional interactive editing may be required.

\subsection{Editing SUMSS Data}

For a project as large as SUMSS, it is important to automate the production of images as much as possible. If interactive editing of data is required, it should be convenient and easily learned. The SUMSS pipeline implements automatic and interactive editing around an image of the entire data set, portrayed as a plot of beam response (greyscale value) in the beam-number plane vs time-scans.

Figure 4 displays the beam-number vs time-scan plot for the field I0331781 (field centre at J0331-78, first observation). Since the MOST uses the principle of Earth rotation synthesis, each full sinusoidal waveform in this plot is the track of a radio source lying in the fan beams during the $12 \mathrm{~h}$ of observation. Tracks that do not lie entirely within the plot throughout the observation are mostly sources lying outside the field of view. These sources will not be fully synthesised. Usually, the contribution of these sources will synthesise to zero within the field of view. Some of the incomplete sinusoidal tracks are grating responses to sources lying in or out of the field. As indicated in Figure 4, an interval of solar interference occurred during this observation, and also there is a relatively strong grating response (grating responses can be recognised by a trained user through their shape and location in the plot).

To remove the artifacts, the entire range of affected time-samples is excluded during synthesis. Figure 5 shows the CLEANed image produced with the range of samples affected by solar interference removed. Compared to

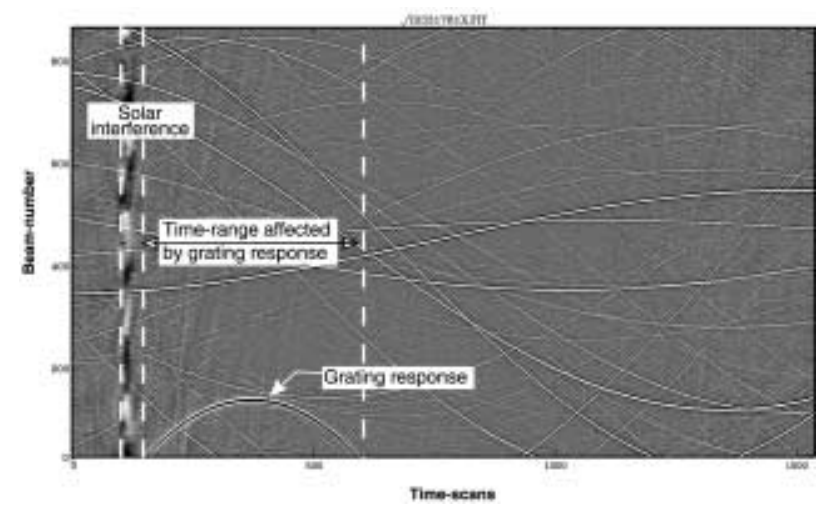

Figure 4 Beam response scan plot of SUMSS field I0331781, corresponding to the image shown in Figure 1. The vertical axis corresponds to the 896 beams, and the horizontal axis shows the 1536 time-samples. The greyscale is proportional to the individual beam flux density at each instant. The indicated time-ranges are the time location of the solar interference (left), and grating response (right). The entire range of time-samples collected during the occurrence of an artifact needs to be excluded from synthesis to eliminate the artifact.

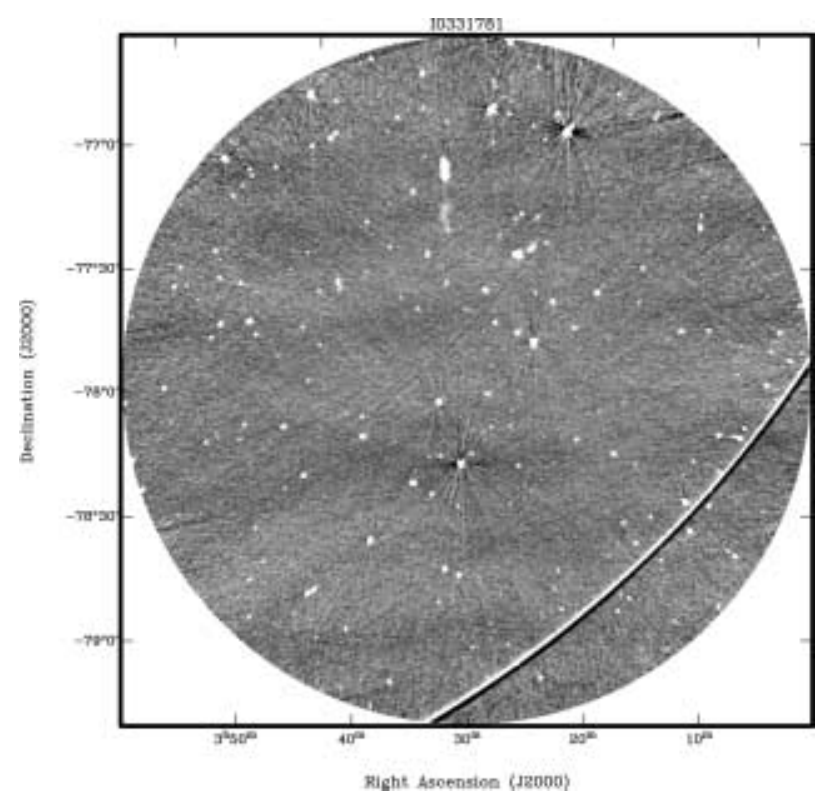

Figure 5 CLEANed image of SUMSS field (I0331781) with samples affected by interference removed, see Section 4.1 . The greyscale is set to -10 to $10 \mathrm{mJy} / \mathrm{beam}$.

Figure 1 (full sample set image), almost all of the bright and dark banding is eliminated. We can also remove the grating response with this method. Figure 6 shows the CLEANed image produced by excluding the range of time-samples affected either by solar interference or the grating response. The grating response and solar banding are eliminated, but the cost is a reduction in image quality owing to the large number of time samples deleted (over 30\%).

However, we can recover much of the lost quality as follows. Employing the MIRIAD task MATHS, we calculate the difference map before and after removing the grating 


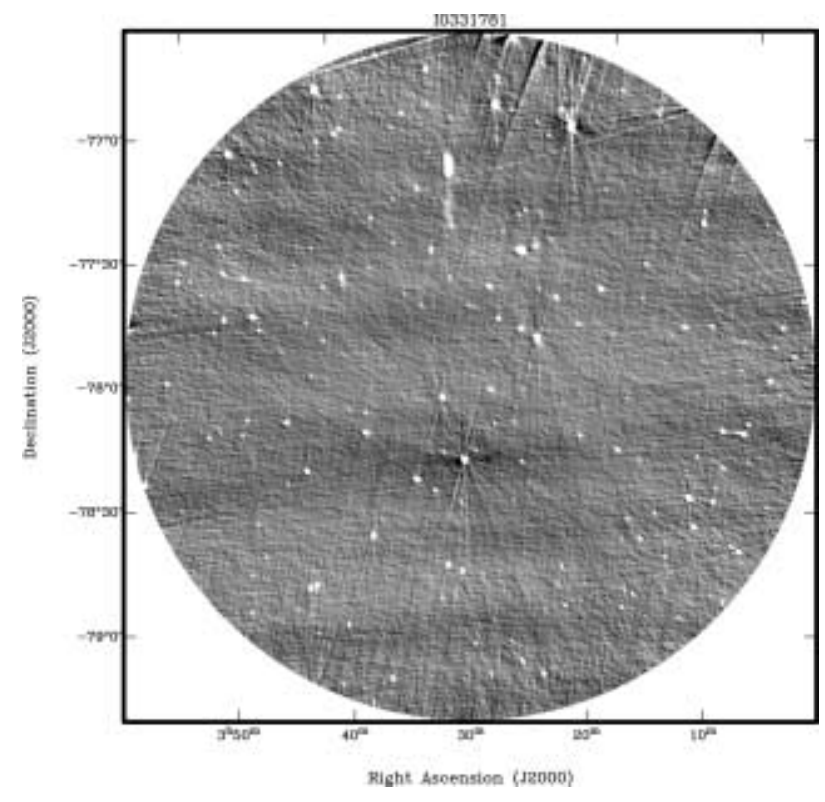

Figure 6 CLEANed image of SUMSS field (I0331781) with samples affected by grating response and interference removed, see Section 4.1. The greyscale is set to -10 to $10 \mathrm{mJy} / \mathrm{beam}$.

response (difference of Figures 5 and 6). This image contains a prominent grating response and little else. With the difference map, using the task IMMASK, we generate a masked subimage excluding the grating response region. This is then added back to the image with the grating response removed (Figure 6). The result (Figure 2) is an image everywhere the same with only solar interference removed (Figure 5), except in the region of the grating response, where it is the lower quality image made with both kinds of artifacts removed (Figure 6).

This method of removing grating responses will not degrade the sensitivity and image quality around sources lying away from the grating response, and the correct flux measurement for previously overlapped sources is now possible.

The deletion of a large range of time samples unfortunately increases the prominence of sidelobes produced by off-field sources which extend into the field of view (marked with boxes in Figure 2). This problem can be fixed in many cases by removing source models obtained from adjacent images, but we are seeking a more convenient approach.

\subsection{Adaptive Deconvolution}

Conventional self-calibration procedures cannot be applied to MOST data, since MOST forms interferometer beams in real time and antenna-based information is not recorded. However, it has been shown that an alternative technique known as adaptive deconvolution may be used (Cram \& Ye 1995). A refined version of the Ye-Cram algorithm has been developed to deal with the 160 arcmin images of the SUMSS survey. Figure 7 shows the CLEANed version of the normal product of the SUMSS analysis pipeline, while Figure 8 shows the image

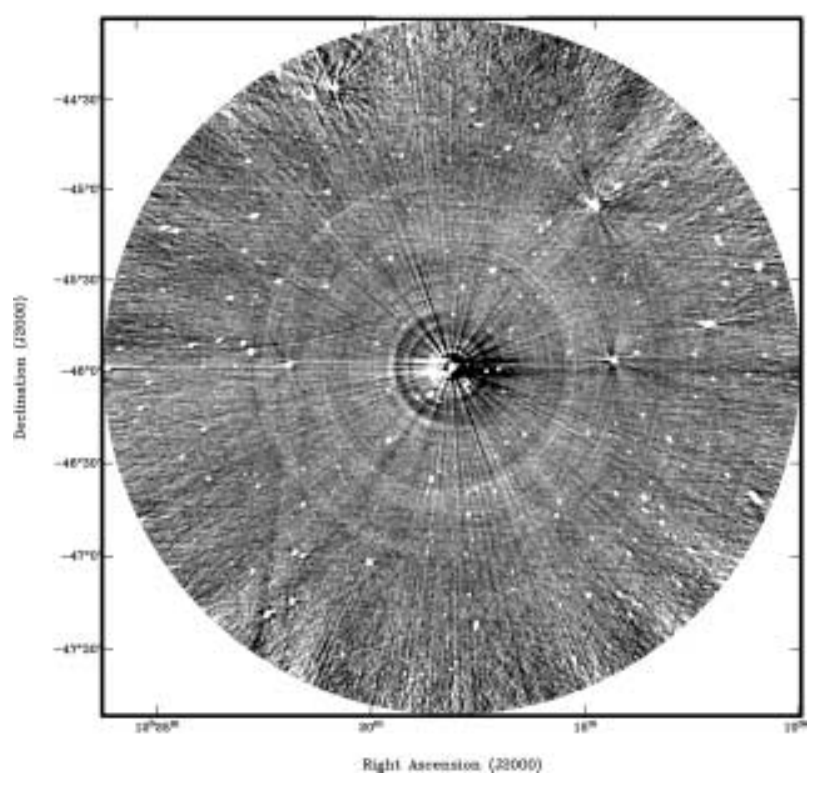

Figure 7 SUMSS field I12154549, with a central strong source at 7.1 Jy. This is the CLEANed image before adaptive deconvolution. The greyscale is set to -10 to $10 \mathrm{mJy} / \mathrm{beam}$.

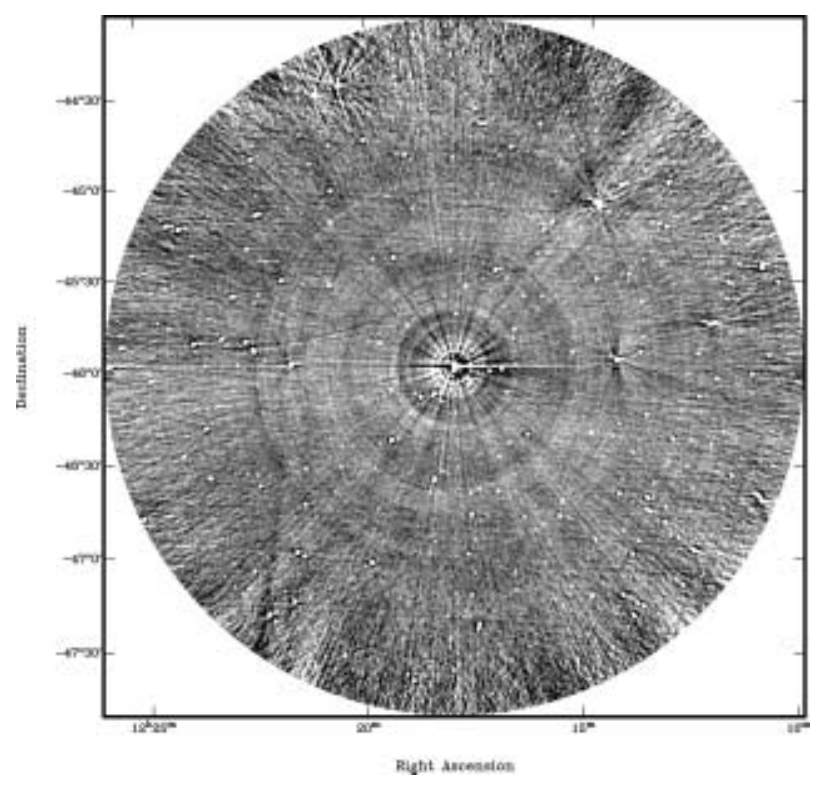

Figure 8 SUMSS field I12154549, with a central strong source at 7.1 Jy. This is the CLEANed image after adaptive deconvolution. The greyscale is set to -10 to $10 \mathrm{mJy} / \mathrm{beam}$.

after adaptive deconvolution. The peak flux density in the image is $7.1 \mathrm{Jy}$, and the greyscale ranges from $-10 \mathrm{mJy}$ to $10 \mathrm{mJy}$. While the adaptive deconvolution algorithm has improved the quality of this extremely demanding field, it would be desirable to devise an even more powerful algorithm and work is under way on this problem.

\section{Application and Conclusion}

We have developed methods to reduce or eliminate interference and grating responses from SUMSS images. We are confident that we can now produce efficiently a source 


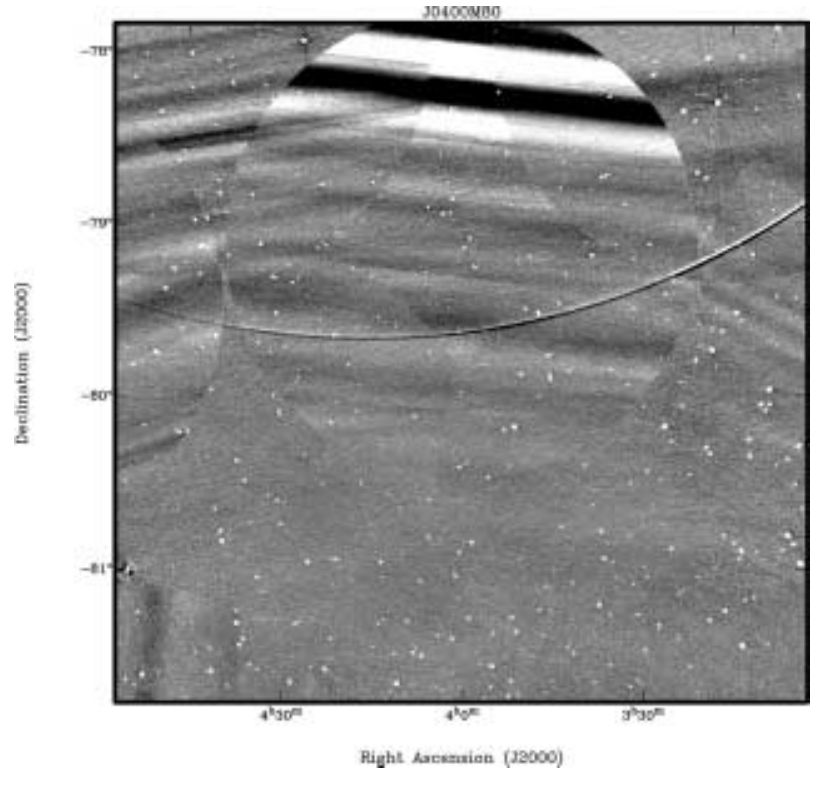

Figure 9 SUMSS mosaic J0400M80, made with individual images without editing, and showing all artifacts. The greyscale is set to -10 to $10 \mathrm{mJy} / \mathrm{beam}$.

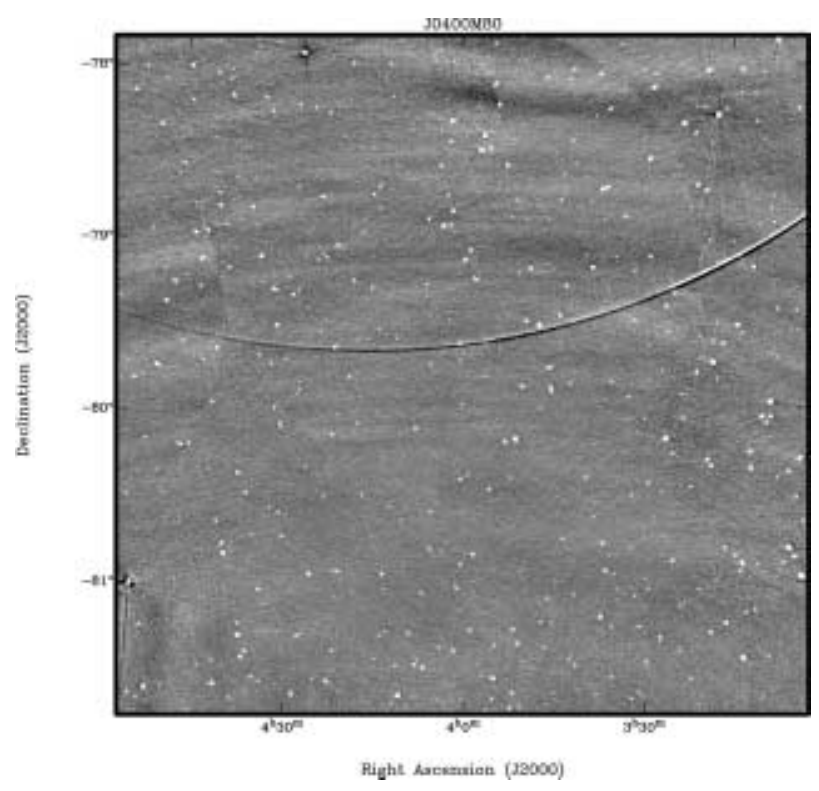

Figure 10 SUMSS mosaic J0400M80, made when individual images have been automatically edited by the standard SUMSS image pipeline. Most solar banding and other interference has been removed, but a strong grating response remains. The greyscale is set to -10 to $10 \mathrm{mJy} / \mathrm{beam}$.

list to a $10 \mathrm{mJy}$ cutoff except in the neighbourhood of very strong sources. Further work on adaptive deconvolution should allow us to work much closer to bright sources.

As an example, Figures 9-11 demonstrate the SUMSS mosaic J0400M80 (mosaic centre at 0400-80, in J2000)

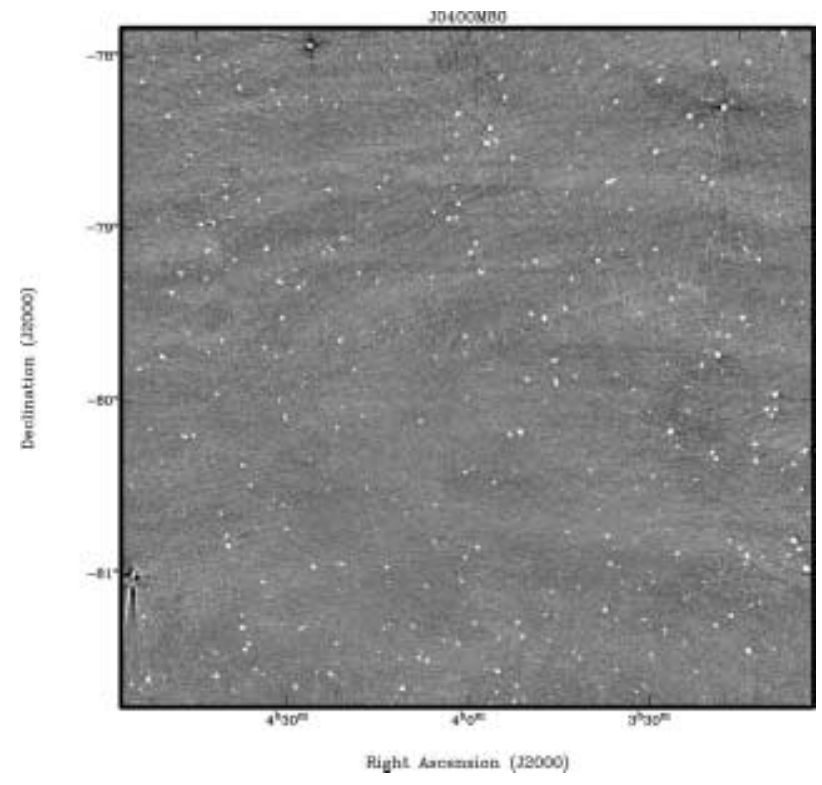

Figure 11 SUMSS mosaic J0400M80, made with individual images after manual editing and removal of grating responses from six of the fields. All severe solar banding and grating responses have been eliminated. The greyscale is set to -10 to $10 \mathrm{mJy} / \mathrm{beam}$.

produced with 10 individual SUMSS fields, without any editing (Figure 9), when individual images have been automatically edited by the standard SUMSS image pipeline (Figure 10), and when the individual images were manually edited for interference and removal of grating responses (Figure 11).

For mosaics such as J0400M80, where radial artifacts are not a major problem (i.e. no sources $>1 \mathrm{Jy}$ ), after removal of interference and grating responses it is relatively easy to produce a source list at $5 \mathrm{mJy}$ cutoff $(\sim 5 \sigma)$. Any spurious fits near strong sources could be easily excised by applying a model of the noise variation as a function of radial distance from the source.

\section{Acknowledgments}

The Molonglo Observatory Synthesis Telescope (MOST) is operated by the University of Sydney and funded by grants from the Australian Research Council. We thank R. Hunstead and E. Sadler for advice regarding the techniques described in this paper.

\section{References}

Bock, D. C.-J., Large, M. I., \& Sadler, E. M. 1999, AJ, 117, 1578

Cram, L. E., \& Ye, T. S. 1995, AuJPh, 48, 113

Large, M. I., Campbell-Wilson, D., Cram, L. E., Davison, R. G., \& Robertson, J. G. 1994, PASA, 11, 44

Mills, B. Y., Little, A. G., \& Joss, G. H. 1976, PASA, 3, 33

Robertson, J. G. 1991, AuJPh, 44, 729 